\title{
MODELAGEM E VALIDAÇÃO DA HIDRATAÇÃO DE GRÃOS DE SOJA
}

Mônica Ronobo COUTINHO', Edilson Sadayuki OMOTO, Cid Marcos Gonçalves ANDRADE ${ }^{3}$, Luiz Mario de Matos JORGE

\section{RESUMO}

Neste trabalho desenvolveu-se um modelo fenomenológico de parâmetros concentrados para a hidratação de grãos de soja, obtido a partir de um balanço de massa em regime transiente nos grãos, levando em consideração a variação do diâmetro destes ao longo do processo. Os resultados das simulações deste modelo foram comparados com as previsões de modelos empíricos encontrados em literatura. Tanto o modelo fenomenológico quanto os modelos empíricos foram validados frente a dados experimentais de hidratação de grãos de soja, obtidos neste trabalho nas temperaturas de $10,20,30,42$ e $49^{\circ} \mathrm{C}$. Todos os modelos representaram as principais tendências do processo de hidratação, a partir do ajuste dos seus parâmetros para cada temperatura. Entretanto, o modelo fenomenológico foi o que apresentou menores desvios em relação aos dados experimentais. Adicionalmente, foi proposto um modelo fenomenológico generalizado.

Palavras-chave: soja; hidratação; modelagem; simulação.

\section{SUMMARY}

MODELING AND VALIDATION OF SOYA BEAN HYDRATION. A first principle model with lumped parameters was developed for the hydration of soya beans. A transient mass balance was applied to the soya beans taking into consideration the variation of the bean diameters during the process. Simulation results were compared to the empirical models available in the literature and with experimental data. Both the first principle and empirical models were validated by the experimental data that were obtained for the temperatures of $10,15,20,30,42$ and $49^{\circ} \mathrm{C}$. All models were able to represent the main data trends of the hydration process, after adjustment of their parameters for each temperature. However, the first principle model has the smallest deviations in relation to the experimental data. In addition, a generalized first principle model was proposed.

Keywords: soya bean; hydration; modeling; simulation.

\section{1 - INTRODUÇÃO}

O processamento da soja freqüentemente requer que os grãos sejam hidratados antes de serem submetidos ao cozimento ou extração de algum constituinte de interesse. Além destas operações, a umidificação também está presente em estudos de secagem de cereais em escala de bancada $[2,5]$ e da capacidade germinativa destes em função do teor de umidade inicial [4].

A umidificação pode ser realizada de diversas maneiras, destacando-se: (a) imersão de grãos em água líquida [3]; (b) exposição dos grãos a uma atmosfera de vapor de água saturado [1, 9]; (c) percolação de um leito de grãos por ar úmido [8].

Uma vez que a absorção de água em grãos depende tanto da temperatura como do tipo de grão, há um grande interesse em caracterizar o comportamento dinâmico do processo de hidratação. Dados de umidade em função do tempo têm sido representados e interpretados por dois tipos básicos de modelos: empíricos e fenomenológicos.

Recebido para publicação em 10/05/2005. Aceito para publicação em 31/08/2005 (001523).

DEALI/UNICENTRO.Email:mcoutinho@unicentro.br

Departamento de Engenharia Quimica Universidade.Estadual de Maringá. Endereço: Campus Universitário, Av. Colombo, 5790, Bloco D90. CEP: 87020-900, Maringá - PR, Brasil. Telefone: (0-xx-44) 32614747, Fax:(0-xx-44) 3261-4792.Email:lmmj@deq.uem.br

A quem a correspondência deve ser enviada.
Os modelos empíricos geralmente são obtidos a partir de simples correlações matemática dos dados experimentais. SINGH \& KULSHRESTHA [12], PELEG [11], SOPADE \& OBEKPA [13] e PAN \& TANGRATANAVALEE [10] são exemplos de pesquisadores que utilizaram modelos empíricos.

SINGH \& KULSHRESTHA [12] desenvolveram um modelo para a curva de absorção de água em grãos de soja (Equação 1), obtendo um ótimo ajuste aos dados experimentais $\left(\mathrm{R}^{2}=0,99\right)$.

$\frac{X_{e}-X}{X_{e}-X_{o}}=\frac{1}{k t+1}$

PELEG [11] propôs um outro modelo empírico (Equação 2) e o ajustou a dados de literatura de absorção de água em leite em pó e arroz, obtendo coeficientes de correlação da ordem de 0,95 a 0,99. Este modelo também foi testado por SOPADE \& OBEKPA [13] para a absorção de água em soja e amendoim, conseguindo representar satisfatoriamente os dados experimentais com coeficiente de correlação equivalente. PAN \& TANGRATANAVALEE [10] usaram o modelo de PELEG [11] com sucesso para estudar a absorção de água por grãos de soja levando em consideração a perda de sólidos.

$X(t)=X_{0}+\frac{t}{100\left(k_{1}+k_{2} t\right)}$

Por sua vez, os modelos fenomenológicos consideram as etapas elementares de transferência de massa. Dentre os estudos desenvolvidos com modelos fenomenológicos, podem-se destacar os trabalhos de HSU [6, 7].

HSU [6] propôs um modelo fenomenológico de parâ- 
metros distribuídos, obtido a partir de um balanço de massa diferencial num grão de soja, admitindo forma esférica de diâmetro constante e representado por uma equação diferencial parcial resolvida numericamente. Posteriormente, HSU [7] estudou a influência da temperatura sobre a difusão de água na soja utilizando o modelo desenvolvido anteriormente, obtendo boa concordância com dados experimentais da hidratação de soja.

Neste trabalho foi desenvolvido um modelo matemático fenomenológico de parâmetros concentrados para a hidratação dos grãos de soja que contempla a variação do volume dos grãos de soja durante o processo. Este modelo foi validado a partir de dados experimentais em várias temperaturas. Adicionalmente, foi comparado com modelos de literatura.

\section{2 - MATERIAL E MÉTODOS}

\section{1 - Procedimento experimental}

O equipamento utilizado neste trabalho consiste de uma caixa de isopor com $39 \mathrm{~cm}$ de comprimento, $25 \mathrm{~cm}$ de largura, $17 \mathrm{~cm}$ de altura e $5 \mathrm{~cm}$ de espessura, ilustrado na Figura 1.

Dentro da caixa foram colocados 2 recipientes com capacidade de 2 litros de água cada. Na parte superior de cada recipiente foi colocada uma peneira, onde se depositavam as amostras de soja com umidade em torno de $10 \%$ (b.u.).

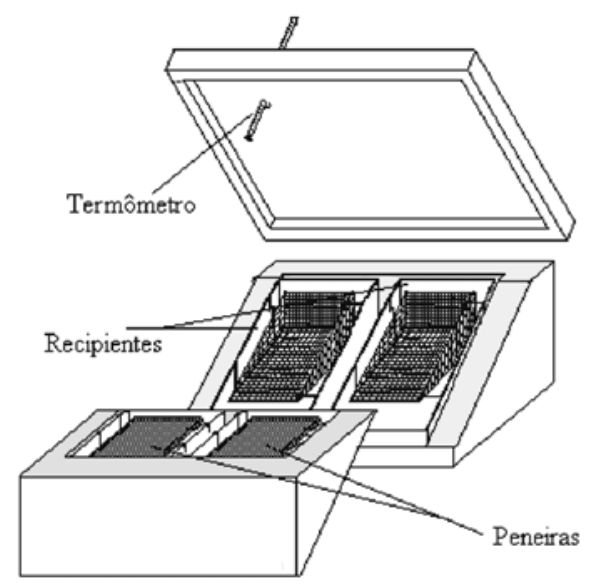

FIGURA 1- Sistema utilizado no experimento

Os experimentos foram realizados conforme seqüência detalhada abaixo:

- colocar água nos recipientes numa determinada temperatura: $10,15,20,30,42$ ou $49^{\circ} \mathrm{C}$;

- adicionar aproximadamente $150 \mathrm{~g}$ de soja em cada peneira;

- acompanhar a hidratação da soja, retirando uma amostra de soja de cada recipiente em intervalos de tempo pré-determinados. Tipicamente nos instantes de tempo: $1,5,10,15,20,25,30,40,50,60,80,100$, 120,150 e $180 \mathrm{~min}$;

- dispor cada amostra de grãos sobre papel toalha, para retirar o excesso de água superficial;

- dividir cada amostra em duas parcelas: a primeira, para determinação do volume e a segunda, para determinação da umidade;

- $\quad$ avaliar o volume e a densidade da soja por picnometria.

- determinar a umidade das amostras pelo método clássico de secagem em estufa a $105^{\circ} \mathrm{C}$ durante $24 \mathrm{~h}$.

\section{2 - Modelagem matemática}

Partindo de um balanço de massa para a água contida na soja e admitindo-se que a concentração de água no grão seja uniforme em cada instante de tempo, obtém-se um modelo de parâmetros concentrados representado pela Equação 3.

$$
\frac{d\left(\rho_{\text {soja }} V\right)}{d t}=K_{s} \cdot A\left(\rho_{H_{2} o}-\rho_{\text {soja }}\right)
$$

Entretanto, ensaios experimentais indicam que o volume do grão de soja varia significativamente durante a hidratação. Logo, a Equação 3 pode ser transformada na Equação 4, contemplando variações do volume e da concentração de água na soja ao longo do tempo.

$$
\rho_{s o j a} \frac{d V}{d t}+V \frac{d \rho_{s o j a}}{d t}=K_{s} A\left(\rho_{H_{2} O}-\rho_{s o j a}\right)
$$

Esta equação pode ser simplificada correlacionando-se experimentalmente a variação do diâmetro dos grãos de soja ao longo do tempo, conforme Equação 5 .

$d_{p}=d_{p_{o}}+0,055 . t^{0,22}$

Admitindo que os grãos de soja sejam esféricos, obtém-se o modelo na sua forma final, conforme Equação 6.

$$
\frac{d \rho_{s o j a}}{d t}=\frac{6 K_{s}\left(\rho_{H_{2} O}-\rho_{s o j a}\right)-\frac{\rho_{s o j a}}{2} 0,0121 . t^{-0,78}}{d_{p_{0}}+0,055 t^{0,22}}
$$

Foram exploradas duas situações distintas: a) $K$ constante; b) $K_{s}$ variando exponencialmente de acordo com a Equação 7.

$$
K_{s}=A_{1} \cdot e^{\left(\beta_{1} \cdot \rho_{s o j a}\right)}
$$

Substituindo-se a Equação 7 na Equação 6, obtémse o modelo com $K_{s}$ variável.

$$
\frac{d \rho_{s o j a}}{d t}=\frac{6 A_{1} \cdot e^{\left(B_{1} \cdot \rho_{s o g}\right)}\left(\rho_{H_{2} O}-\rho_{s o j a}\right)-\frac{\rho_{s o j a}}{2} 0,0121 \cdot t^{-0,78}}{d_{p_{0}}+0,055 t^{0,22}}
$$


Apesar de apresentarem-se os modelos em função de concentração de água na soja $\left(\rho_{s_{\text {o ja }}}\right)$ é mais usual utilizarse a umidade em base seca $\left(\mathrm{X}_{\mathrm{s}}\right)$. Esta conversão pode ser feita através da Equação 9.

$X_{S}=\frac{\rho_{\text {soja }}}{D_{\text {soja }}-\rho_{\text {soja }}}$

\section{3 - Ajuste individual dos modelos matemáticos}

O modelo de PELEG [11] possui dois parâmetros: $k$ e $k$ Estes foram obtidos a partir da linearização do modelo e ajuste individual aos dados experimentais por regressões lineares, enquanto os parâmetros do modelo de SINGH \& KULSHRESTHA [12]: $k$ e $X$ foram obtidos a partir de regressões não-lineares efetuadas no programa STATISTICA 5.1.

Os parâmetros do modelo fenomenológico: $K_{s}, A_{i}$ e $B$, foram obtidos através de integração numérica da Equação 6 ( $K_{s}$ constante) ou Equação 8 ( $K_{s}$ exponencial) com a condição inicial: $\mathrm{t}=0, \rho_{\text {so }}=\rho_{\mathrm{s}}$, no programa MATLAB (versão 5.1), seguido de ajuste pelo método dos mínimos quadrados, utilizando a rotina fmins.m para minimizar o resíduo quadrático: $\phi^{2}=\sum\left(X_{c a t}-X_{\ldots, p}\right)^{2}$.

\section{3 - RESULTADOS E DISCUSSÃO}

\section{1 - Simulações a partir de parâmetros de lite- ratura}

Na Figura 2, observa-se que as curvas de hidratação previstas pelo modelo de SINGH \& KULSHRESTHA [12] com parâmetros apresentados pelos autores em temperaturas entre 37 e $65^{\circ} \mathrm{C}$ não representam adequadamente os resultados experimentais obtidos neste trabalho, apesar de reproduzir as principais tendências.

Na Figura 3 estão representadas as curvas de hidratação previstas pelo modelo de PELEG com parâmetros obtidos por SOPADE \& OBEKPA [13] nas temperaturas de 2,25 e $40^{\circ} \mathrm{C}$ e por PAN \& TANGRATANAVALEE [10] nas temperaturas de $10,20,30$ e $40^{\circ} \mathrm{C}$.

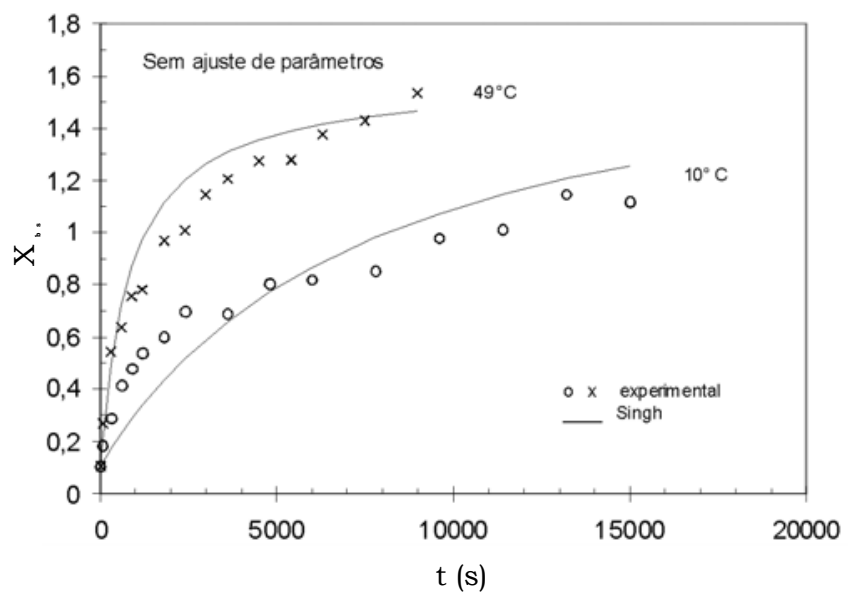

FIGURA 2 - Comparação do modelo de SINGH \& KULSHRESTHA [12] frente a dados experimentais

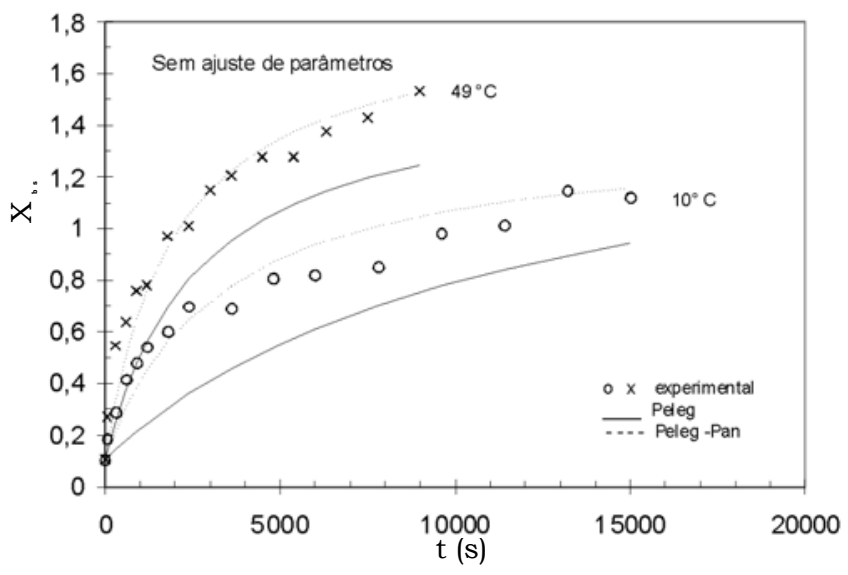

FIGURA 3 - Comparação do modelo de PELEG frente a dados experimentais 
Observa-se na Figura 3 acima que o modelo de PELEG [11] também não representa quantitativamente os resultados experimentais, porém, as principais tendências são reproduzidas. Os parâmetros obtidos por PAN \& TANGRATANAVALEE [10] conduzem a uma melhor representação dos resultados experimentais do que os parâmetros obtidos por SOPADE \& OBEKPA [13].

\section{2 - Variação do diâmetro ao longo da hidratação}

O estudo da variação do diâmetro, ao longo da hidratação, realizado nas temperaturas de 10, 15, 20, 30, 42 e $49^{\circ} \mathrm{C}$, mostrou que a temperatura não exerce uma influência significativa sobre o comportamento do diâmetro ao longo do tempo conforme se pode observar na Figura 4. Desta forma, foi proposta uma correlação de d em função do tempo, independente da temperatura, obtida por ajuste dos dados experimentais. Observa-se que são nos primeiros 15 minutos que o aumento do diâmetro é mais significativo $( \pm 15 \%)$ e que ao final da hidratação o diâmetro aumenta aproximadamente 30\%. Este aumento de $30 \%$ mostra que a variação de volume ao longo da hidratação é significativa.

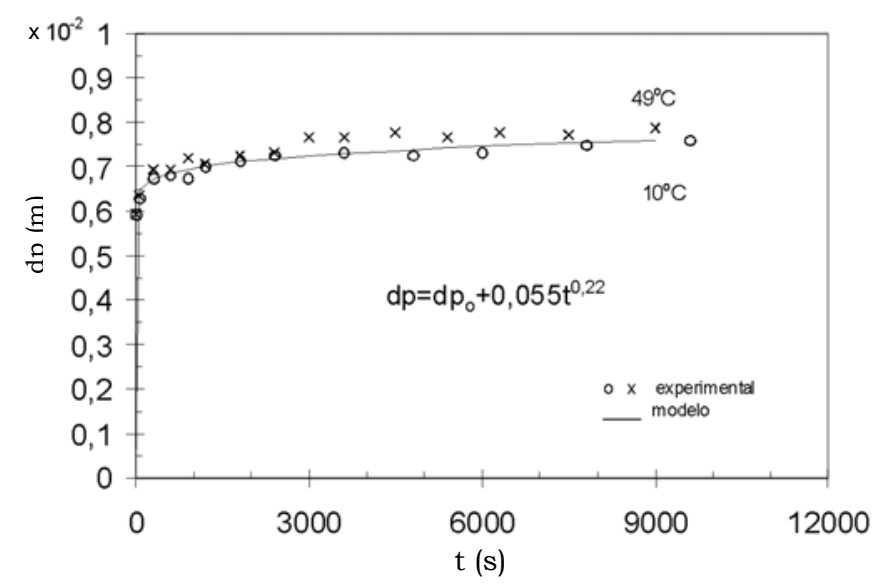

FIGURA 4 - Comportamento do diâmetro da soja ao longo da hidratação

\section{3 - Ajuste individual dos modelos matemáti- $\cos$}

$K_{s}$ constante: Como pode-se observar na Figura 5 o modelo com $K_{s}$ constante mostrou-se inadequado para representar o processo de hidratação.

$K_{s}$ variável: A Figura 6 apresenta os resultados das si- mulações do modelo fenomenológico com $K_{s}$ variando exponencialmente juntamente com valores experimentais e com as previsões dos modelos de SINGH \& KULSHRESTHA [12] e de PELEG [11] com parâmetros ajustados. Como se pode observar, os modelos ajustados individualmente apresentam tendência semelhante, porém com resultados diferenciados entre si.

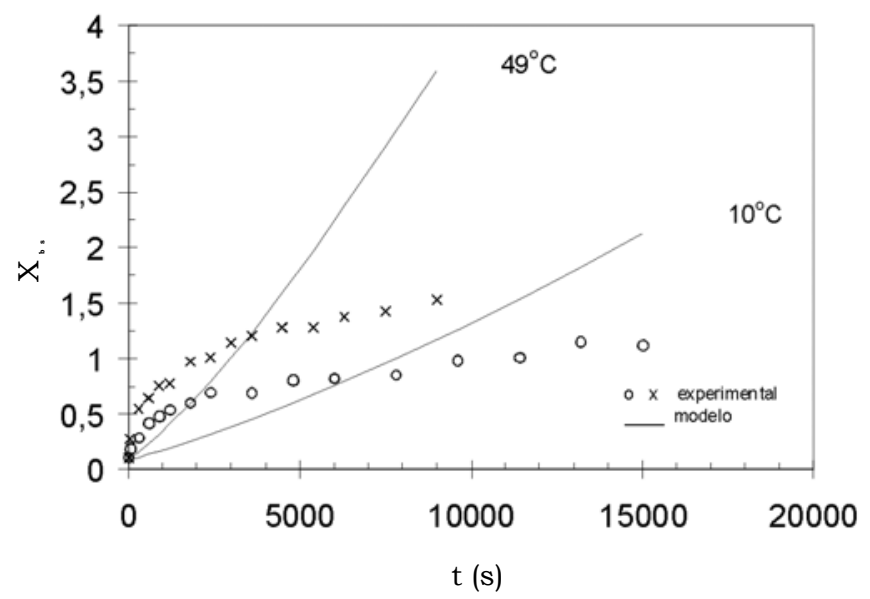

FIGURA 5 - Previsões do modelo com $K_{s}$ constante 


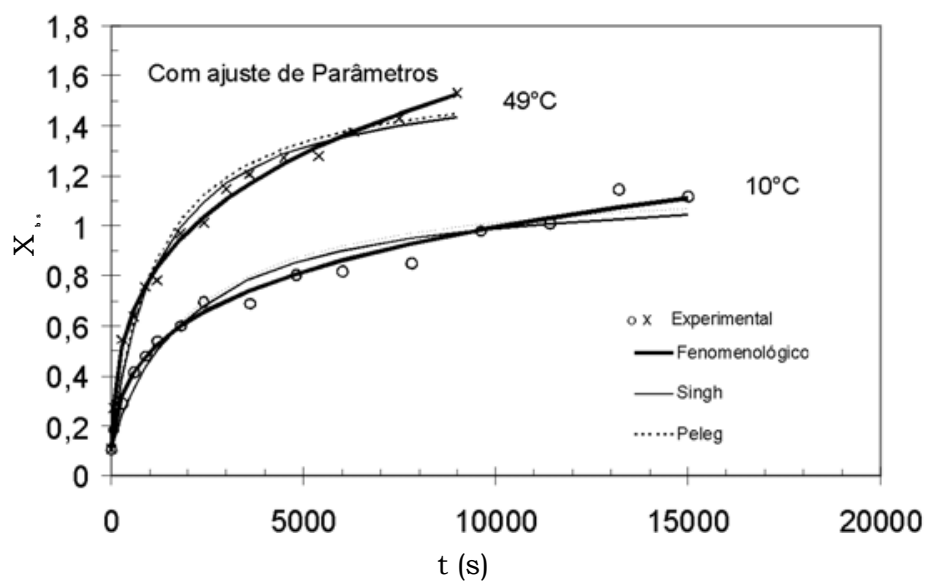

FIGURA 6 - Previsões dos modelos empíricos e fenomenológico frente a dados experimentais

Apesar dos modelos de SINGH \& KULSHRESTHA [12] e de PELEG [11] representarem as principais tendências da hidratação, constata-se que o modelo fenomenológico apresentou o menor resíduo quadrático $\left(\phi^{2}=0,17\right)$, quando comparado aos modelos empíricos de SINGH \& KULSHRESTHA $\left(\phi^{2}=0,46\right)$ e de PELEG $\left(\phi^{2}=0,50\right)$, como se pode observar nas Figuras 7,8 e 9. Também é possivel notar que os modelos empíricos apresentam maior erro no início da umidificação, correspondendo aos menores valores de $X_{s,}$ (Figura 7).

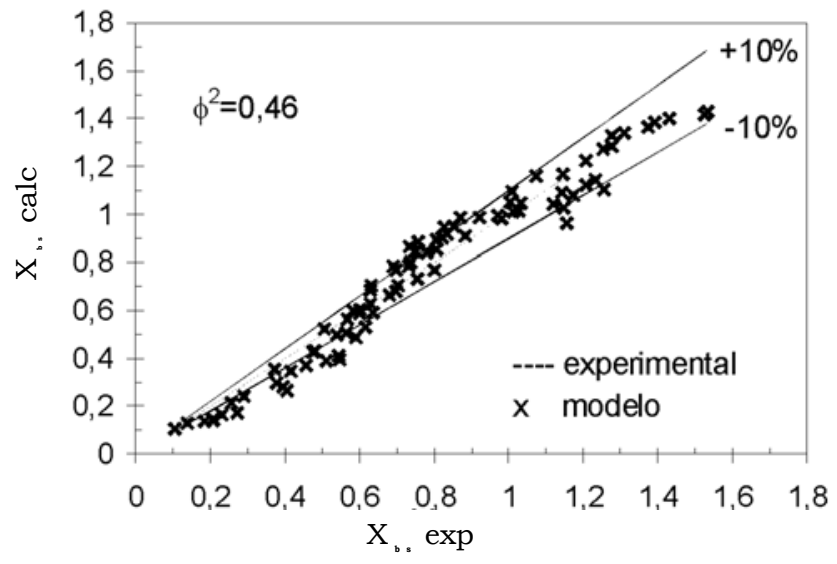

FIGURA 7 - Desvios do modelo de SINGH \& KULSHRESTHA [12]

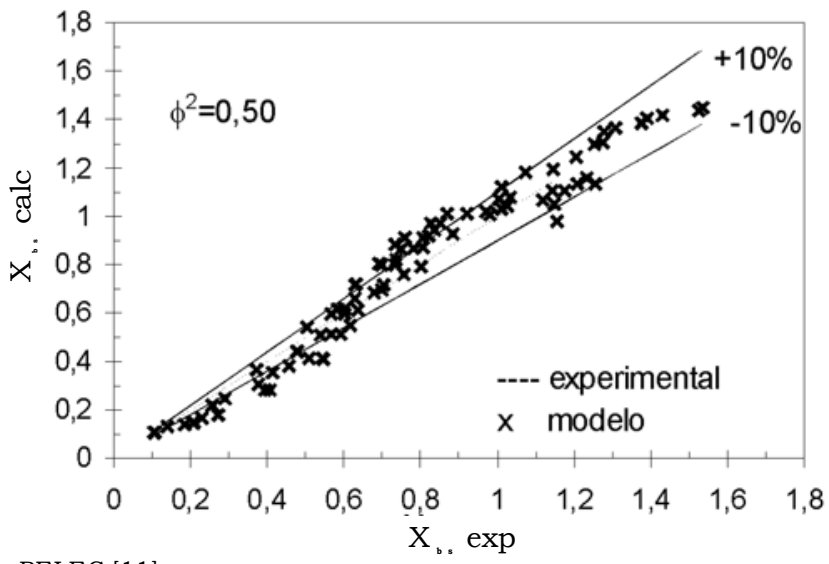

FIGURA 8 - Desvios do modelo de PELEG [11] 
Modelagem e validação da hidratação de grãos de soja, Coutinho et al.

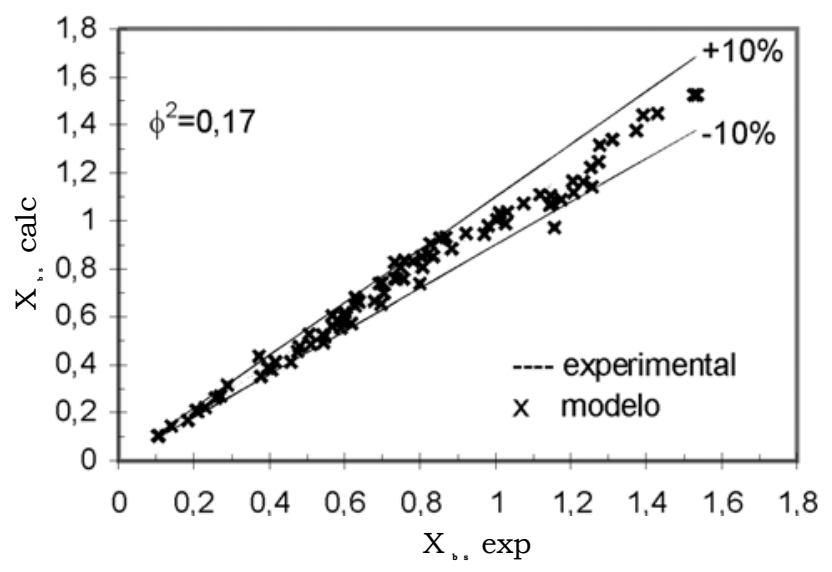

FIGURA 9 - Desvios do modelo fenomenológico

3.4 - Influência da temperatura sobre os parâmetros do modelo fenômenológico

Todos os parâmetros do modelo fenomenológico com $K_{s}$ variável foram correlacionados em função da temperatura, conforme Figuras 10 e 11. Na Figura 10, observa-se que o parâmetro $A$ do coeficiente aparente de transferência de massa $\left(K_{s}\right)$ tende a aumentar linearmente com a temperatura, ao contrário do parâmetro $B$ que é praticamente constante e pode ser representado por um valor médio $\left(\bar{B}=-8,40 \times 10^{3}\right)$, conforme Figura 11 .

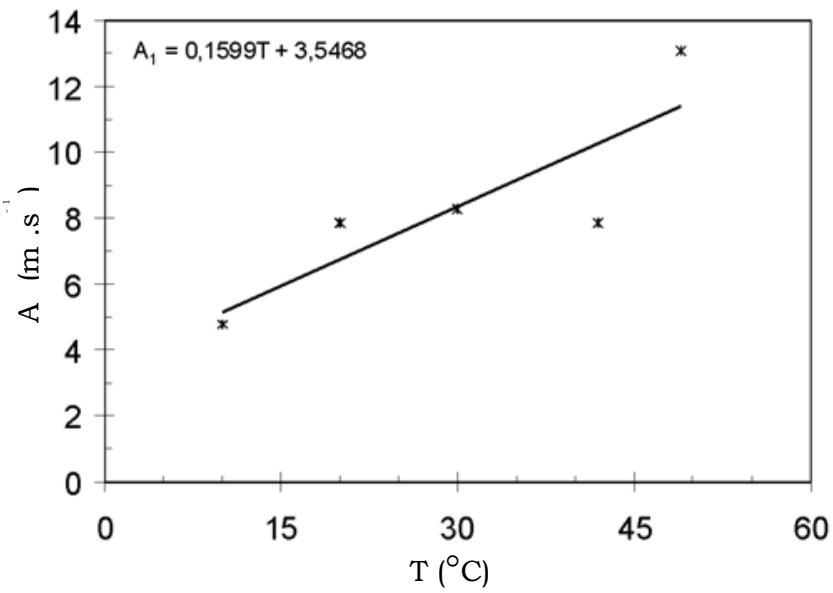

FIGURA 10 - Influência da temperatura no parâmetro $A_{\text {s }}$

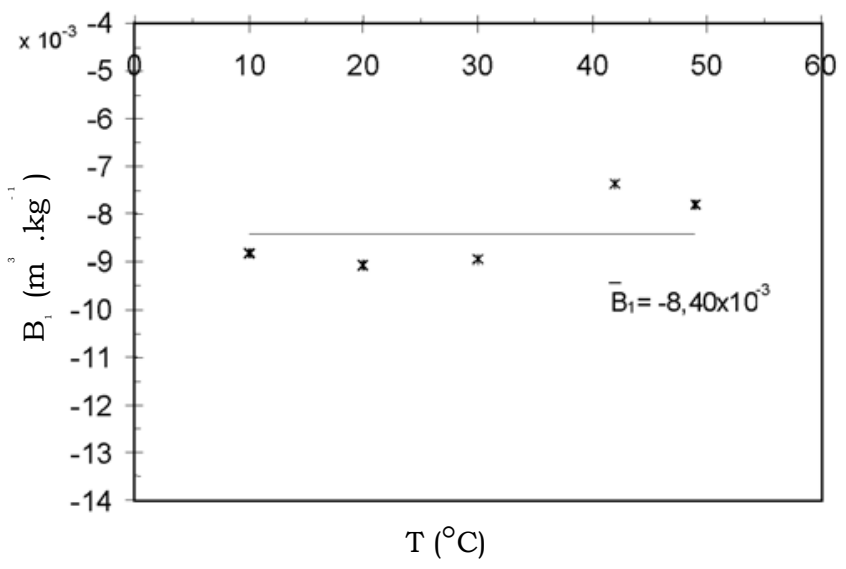

FIGURA 11 - Influência da temperatura no parâmetro $B$ 


\section{5 - Modelo fenomenológico generalizado}

Com a correlação obtida de $A$ em função da temperatura e o valor médio de $B$, apresentadas nas Figuras 10 e 11, generalizou-se o modelo fenomenológico, Equação 9, simulando-o em todas as condições experimentais. Na Figura 12 pode-se observar que o modelo generalizado não representa os valores experimentais tão bem quanto o modelo ajustado, porém, prevê satisfatoriamente o comportamento da curva de hidratação.
Na Figura 13 são comparadas todas as simulações com todos os dados experimentais de umidade, revelando que o desvio quadrático apresentado pelo modelo fenomenológico generalizado $\left(\phi^{2}=0,86\right)$ é maior do que o do modelo fenomenológico com parâmetros ajustados individualmente $\left(\phi^{2}=0,17\right)$, como esperado. Entretanto as previsões apresentam um erro predominante na faixa de 0 a $20 \%$ em relação aos valores experimentais revelando que o modelo generalizado pode ser utilizado satisfatoriamente como uma primeira estimativa.

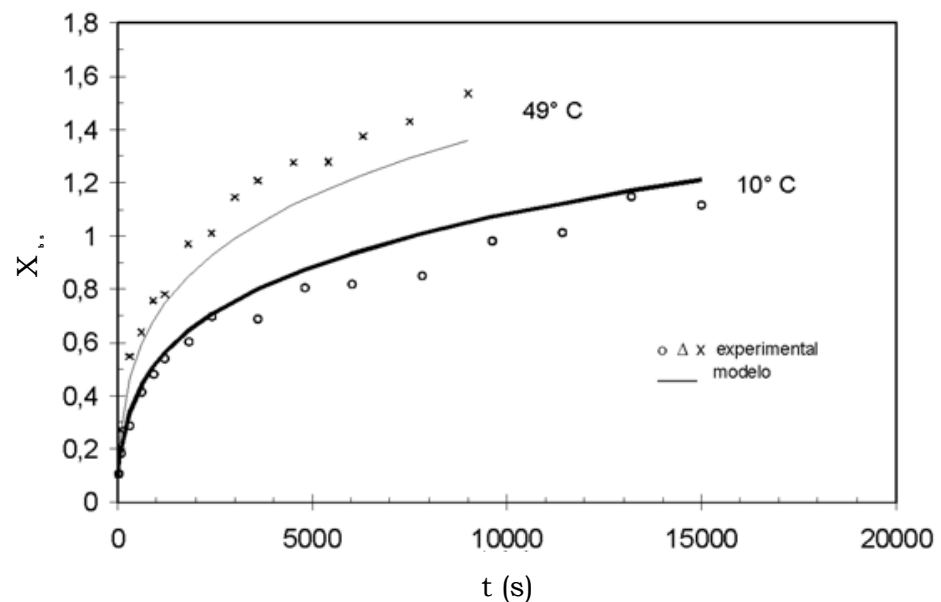

FIGURA 12 - Previsões do modelo generalizado

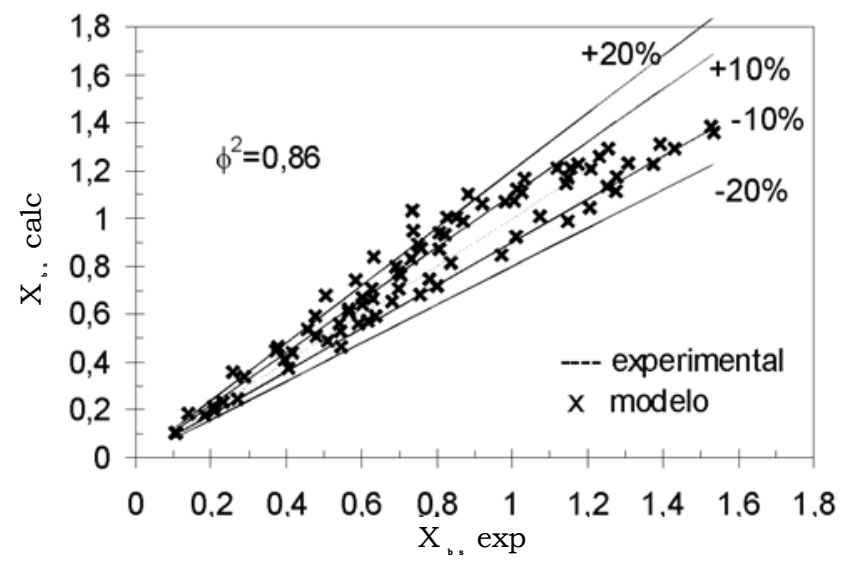

FIGURA 13 - Desvios do modelo fenomenológico generalizado

\begin{tabular}{|c|c|c|c|c|}
\hline \multicolumn{2}{|c|}{3.6 - Nomenclatura } & $t$ & tempo & (s) \\
\hline$A$ & Área & $T$ & temperatura & $\left({ }^{\circ} \mathrm{C}\right)$ \\
\hline$A_{i}$ & parâmetro de ajuste do modelo Fenomenológico (adm.) & $V$ & volume do grão & $\left(\mathrm{m}^{3}\right)$ \\
\hline$B$ & parâmetro de ajuste do modelo Fenomenológico (m.s ) & $X_{s}$ & umidade em base seca & (adm.) \\
\hline$d_{0}$ & diâmetro médio da soja & $X_{0}$ & umidade de equilíbrio em base seca & (adm.) \\
\hline$d$ & diâmetro inicial da soja & $X_{o}$ & umidade inicial em base seca & (adm.) \\
\hline$k$ & constante de Peleg & $\rho_{\mathrm{H}_{2}}$ 。 & densidade da água & $\left(\mathrm{kg} \cdot \mathrm{m}^{-3}\right)$ \\
\hline$k_{2}$ & constante de Peleg & $\rho_{s o j a}^{2}$ & concentração da água na soja & $\left(\mathrm{kg} \cdot \mathrm{m}^{-3}\right)$ \\
\hline 1 & coeficiente aparente de transferência de massa (m.s ) & $\phi^{2}$ & desvio quadrático & (adm.) \\
\hline
\end{tabular}




\section{4 - CONCLUSÕES}

Todos os modelos puderam representar as principais tendências do processo de hidratação a partir do ajuste dos seus parâmetros. Entretanto, o modelo fenomenológico foi o que melhor representou a hidratação de grãos de soja em toda a faixa de temperatura explorada $\left(10 \mathrm{a} 49^{\circ} \mathrm{C}\right)$.

O diâmetro dos grãos de soja apresentou um aumento médio em torno de $30 \%$ ao final de 150 minutos.

A maior taxa de variação de diâmetro dos grãos de soja ocorreu nos primeiros 15 minutos, correspondendo a $50 \%$ do valor final.

A temperatura não exerce influência significativa sobre o comportamento do diâmetro do grão, ao longo do tempo na faixa de temperaturas exploradas.

O coeficiente aparente de transferência de massa $(k)$ varia ao longo da hidratação e pôde ser representado satisfatoriamente por uma função exponencial: $K_{s}=A_{1} \cdot e^{\left(B_{1} \cdot P_{\text {roje }}\right)}$

As previsões do modelo fenomenológico generalizado apresentaram um erro na faixa de 0 a $20 \%$ em relação aos valores experimentais, podendo ser utilizado satisfatoriamente como uma primeira estimativa.

\section{5 - REFERÊNCIAS BIBLIOGRÁFICAS}

[1] AGUERRE, R.; SUAREZ, C.; VIOLLAZ, P.E. Drying Kinetics of Rough Rice Grain. J. Food Technol., v. 17, p. 679-686, 1982.

[2] BARROZO, M. A. S; CALADO, V.M.A.; CALÇADA, L.A.; MASSARANI, G. Secagem de Soja em Leito Deslizante e Recirculação Pneumática: Influência da Umidificação Artificial, v. I, p. 327-337, Campinas, SP, 1991.

[3] BECKER, H. A.; SALLANS, H. R. A Study of Diffusion in Solids of Arbitrary Shape, with Application to the Drying of the Wheat Kernel. Cereal Chemistry, v. 32, p. 212226,1955
[4] BECKERT, O. P; MIGUEL, M.H.; MARCOS FILHO, J. Absorção de Água e Potencial Fisiológico em Sementes de Soja de Diferentes Tamanhos. Scientia Agrícola, v. 57, n. 4, p. 671-675, 2000.

[5] CALADO, V. M. A. Modelagem e Simulação de Secadores em Leitos Fixo e Deslizante. 1993, Tese D.Sc., COPPE, UFRJ.

[6] HSU, K. H. A Diffusion Model with a ConcentrationDependent Diffusion Coefficient for Describing Water Movement in Legumes During Soaking. J. of Food Sci. v. 48, p. 618-622 e $645,1983$.

[7] HSU, K. H. Effect of Temperature on Water Diffusion in Soybean. J. of Food Sci., v. 48, p. 1364 e 1365, 1983.

[8] MANTOVANI , B. H. M. Análise e Simulação de Secagem de Grãos de Milho em Camadas Espessa. Viçosa, MG, 1976. Dissertação de M.Sc., Departamento de Engenharia Agrícola, Universidade Federal de Viçosa.

[9] NOVAIS, A. F. Análise da Aplicação do Leito Deslizante e Escoamentos Cruzados na Secagem de Sementes. São Carlos, SP, 1990. Dissertação M.Sc. PPG-EQ, Universidade Federal de São Carlos.

[10] PAN, Z.; TANGRATANAVALEE, W. Characteristics of soybean as affected by soaking conditions, Lebensm. Wiss. U.- Technol., v.36, p. 143-151, 2003.

[11] PELEG, M. An Empirical Model for the Description of Moisture Sorption Curves. J. of Food Sci., v. 53, n. 4, p. 1216-1217 e 1219,1988

[12] SINGH, B. P. N.; KULSHRESTHA, S. P. Kinetcs of Water Sorption by Soybean and Pigeonpea Grains. J. of Food Sci., v. 52, n. 6, p. 1538-1541 e 1544, 1987.

[13] SOPADE, P. A.; OBEKPA, J.A. Modelling Water Absorption in Soybean, Cowpea and Peanuts at Three Temperatures Using Peleg's Equation. J. of Food Sci., v. 55, n. 4 , p. $1085-1087,1990$ 